\title{
El sujeto discursivo: la construcción social de subjetividades en el pensamiento de Bajtín y su Círculo
}

\author{
Erika Lindig Cisneros
}

Este trabajo pretende discutir la reelaboración crítica de las nociones de "sujeto" y "conciencia" en algunos textos de Mijaíl Bajtín y sus colaboradores. Si Bajtín y los miembros de su Círculo de estudios heredaron del romanticismo alemán, y específicamente de W. von Humboldt la tesis de que la conciencia se constituye lingüísticamente (aunque no necesariamente reconozcan esta herencia), la concepción general del lenguaje como fenómeno socio-ideológico modifica las nociones de conciencia y sujeto que se constituyen en el medio lingüístico. Me interesa reflexionar sobre las posibilidades teóricas que se abren a partir de esta modificación, y en su vigencia para pensar la problemática de las exclusiones sociales en el ámbito del discurso.

Palabras clave: Bajtín, sujeto, conciencia, discurso, lenguaje.

This paper discusses the critical re-signification of the notions "subject" and "consciousness" in some works of Mikhail Bakhtin and his circle. If Bakhtin and the members of his circle inherited from German Romanticism, and specifically from Wilhelm von Humboldt, the tenet that consciousness is linguistically constituted (even if they did not fully acknowledge this influence), the recognition of language as a socio-ideological phenomenon, not present in Humboldt's Romanticism, modified the notions of consciousness and subject, which depend on language. I dwell on the theoretical possibilities arising from this modification and on their relevancy when thinking about the issues of discursive social exclusion.

KEY WORDs: Bakhtin, subject, consciousness, discourse, language. 

Erika Lindig Cisneros

Facultad de Filosofía y Letras,

Universidad Nacional Autónoma de México

\section{El sujeto discursivo: la construcción social de subjetividades en el pensamiento de Bajtín y su Círculo}

El debate contemporáneo sobre los problemas de exclusión en nuestras sociedades llamadas "democráticas" se enfrenta a un problema terminológico: ${ }^{1}$ la necesidad de usar el término "sujeto" tras el cuestionamiento del mismo, iniciado por Nietzsche desde la segunda mitad del siglo XIX y reelaborado por muy distintas posturas teóricas durante todo el siglo pasado. Esta necesidad de seguir hablando de sujetos puede explicarse desde la práctica jurídica, que aún se funda en una concepción del sujeto del derecho como sujeto "libre", en el sentido de ser dueño de su propia voluntad y por tanto capaz de tomar decisiones. Pero de manera más interesante, la necesidad de hablar de sujetos, ya sea individuales o colectivos, acabados o en proceso de construcción, puede explicarse también desde el discurso

\footnotetext{
${ }^{1}$ Pero no un mero problema terminológico. La terminología o el vocabulario es fundamental desde el momento en que es objeto de apropiación por parte de todo tipo de fuerzas sociales y políticas, y además porque puede pensarse como ese lugar donde es posible el intercambio entre las diversas disciplinas y también entre teorías disciplinares y las referencias, por ejemplo, entre las humanidades y los movimientos sociales (Martínez de la Escalera, "Prólogo", 7-9).
} 
de las víctimas de las diversas formas de exclusión inherentes a nuestros sistemas políticos (o policiacos, en el sentido de Rancière). ${ }^{2}$ Estas víctimas, cuando se organizan en movimientos de resistencia, apelan a la construcción de "identidades" y "subjetividades" alternativas precisamente para resistir a las prácticas hegemónicas excluyentes. Un ejemplo interesante de resistencia en este sentido es el de las Misrahi: un grupo de mujeres israelíes, judías de origen oriental, quienes se autodefinen como "Feministas Misrahi". Sigal Nagar-Ron, socióloga e investigadora de la Universidad Ben Gurión, lo describe como un grupo doblemente excluido de la sociedad israelí: tanto desde el punto de vista de su origen étnico como por su género. Ella trabajó a partir de los testimonios de algunas de estas mujeres (académicas en su mayoría), quienes además de luchar contra la opresión hacia su grupo particular trabajan solidariamente con otras organizaciones sociales. En el análisis de los testimonios Nagar-Ron sostiene que, morfológicamente, todas las historias de vida de estas mujeres revelan estructuras similares. La autobiografía se relata como una historia en dos etapas: la primera se define en cada caso como un periodo en el cual aún no se ha desarrollado todavía una conciencia étnica, y la segunda como aquella en la cual sobreviene el desarrollo de dicha conciencia:

En la primera etapa internalizaron las percepciones hegemónicas reinantes en la sociedad y pretendieron desarrollar una identidad que se ajustara a las demandas ideológicas de la hegemonía. La segunda etapa se relaciona con el proceso median-

\footnotetext{
2 "Policía u orden policial (La Police or L’Ordre policier). La policía, entendida como la ley general que determina la distribución de partes en una comunidad, así como sus formas de exclusión, es en primer lugar y sobre todo una organización de 'cuerpos, fundada en una distribución común de lo sensible, esto es, un sistema de coordenadas que determinan modos de ser, hacer, y comunicar que establecen las fronteras entre lo visible y lo invisible, lo audible y lo inaudible, lo decible y lo indecible" (Rancière, The Politics of Aesthetics, 89).
} 
te el cual se percataron de su opresión como parte de un grupo étnico excluido, y con los cambios que tuvieron lugar en su autoconciencia y en su identidad a la luz de esta nueva conciencia (Nagar-Ron, "Identidad, resistencia y voz", 17).

Como puede verse, Nagar-Ron recurre a los términos "conciencia" e "identidad", y en otros fragmentos a "sujetos", además de "sujetos obedientes" — en el sentido de Foucault — para analizar el cambio que se produjo en la concepción de sí misma de cada una de estas mujeres, y que les abrió la posibilidad de organizarse y ofrecer resistencia a las diversas formas de exclusión. Las prácticas de subjetivación mediante las cuales estas mujeres elaboran estrategias de resistencia son prácticas discursivas (Nagar-Ron, "Identidad, resistencia y voz", 15-20). La teoría bajtiniana del discurso, en su discusión con el romanticismo alemán, puede aportar un concepto de sujeto todavía vigente hoy para pensar en estos casos de exclusión sociopolítica y en los procesos de subjetivación necesarios para cuestionar el orden que produce las exclusiones.

\section{Conciencia y sujeto}

En El marxismo y la filosofía del lenguaje aparece la siguiente afirmación: "La conciencia individual es un hecho ideológico y social. Hasta que este postulado se reconozca con todas sus implicaciones, no podrá construirse una psicología objetiva ni una ciencia objetiva de las ideologías" (Voloshinov, Marxismo y filosofía del lenguaje, 35-36). Para interpretarla es necesario recordar que según Voloshinov / Bajtín ${ }^{3}$ el terreno de la ideolo-

\footnotetext{
${ }^{3}$ No voy a entrar aquí en la discusión sobre la autoría de los textos publicados por los miembros del "Círculo Bajtín". Es pertinente anotar, sin embargo, que dicha discusión sigue vigente para efectos interpretativos. Remito al lector al artículo de Tatiana Bubnova "Bajtín en la encrucijada dialógica (datos y comentarios para
} 
gía coincide con el de los signos, ${ }^{4}$ y que la palabra ${ }^{5}$ es el signo ideológico por excelencia, entre otras razones, por servir como medio para la conciencia. Tenemos, pues, que la conciencia de los sujetos individuales es, en primer lugar, una conciencia "estructurada verbalmente", o bien una conciencia linguiística.

Esta tesis, que afirma la imposibilidad de pensar en la conciencia y en su actividad fuera del lenguaje, es - como sabemosherencia del romanticismo alemán, y particularmente de la filosofía del lenguaje de Wilhelm von Humboldt. En El marxismo y la filosofía del lenguaje, Humboldt aparece como el mayor representante de una de las dos grandes tendencias del pensamiento filosófico-lingüístico, el subjetivismo individualista, aunque su filosofía del lenguaje se reconozca lo suficientemente compleja y contradictoria para rebasar la caracterización de dicha tendencia. Anecdóticamente, es notable la coincidencia entre la evaluación que Bajtín hace de ella y la realizada pos-

contribuir a la confusión general)", y en general a Zavala (coord.), Bajtín y sus apócrifos, en donde aparece este artículo.

${ }^{4}$ En este texto, Voloshinov / Bajtín se refiere al "signo" como cualquier entidad material que "refleja y refracta" una realidad distinta de sí misma, y a la "ideología" como proceso general de producción de significación. Sobre esta última noción, M. Gardiner indica que si bien no hay en el texto una definición, es posible, con una lectura cuidadosa, dibujar la concepción que Voloshinov / Bajtín pergeñaron. Ellos, lejos de interpretar el término "ideología" como "falsa conciencia" o como un "conjunto coherente de creencias", la interpretan como el medio simbólico a través del cual todas las relaciones sociales necesariamente se constituyen (Gardiner, M.M. Bakhtin and the Theory of Ideology, 6-7).

${ }^{5}$ El término que utiliza Bajtín para referirse al objeto de sus problematizaciones sobre el lenguaje es el vocablo ruso "slovo". En la traducción al castellano de $E l$ marxismo y la filosofía del lenguaje, Tatiana Bubnova anota que dicho vocablo cubre un campo semántico amplio, que sólo en parte coincide con el castellano, y permite un permanente juego conceptual. Ella se remite al Diccionario de la lengua rusa de S.I. Ozhegov, que ofrece nueve acepciones, de las cuales interesan las siguientes: a) unidad de la lengua que sirve para nombrar un concepto aislado; b) el mismo discurso, la facultad de hablar; c) actuación pública oral, discurso en una asamblea; d) discurso sobre algún tema, narración, exposición (arcaísmo de sentido elevado); e) opinión, alegato (Cfr. Voloshinov, Marxismo y filosofía del lenguaje, 37). 
teriormente por Heidegger: ambas consideran que la filosofía del lenguaje de Humboldt determina toda la lingüística y la filosofía del lenguaje posteriores, ${ }^{6}$ lo cual es probablemente una exageración. Más importante desde el punto de vista teórico es que ambas coinciden en cuestionar la filosofía del lenguaje de Humboldt por su énfasis en el sujeto de conocimiento como punto de partida para la reflexión sobre el lenguaje.

De acuerdo con Heidegger, Humboldt no logró salir del esquema moderno de conocimiento sujeto-objeto. Interpretando un fragmento de Humboldt sobre el lenguaje, no como simple medio de intercambio para la comprensión recíproca sino como un verdadero mundo que el espíritu debe poner entre sí y los objetos a través de la labor interna de su fuerza, afirma que:

De acuerdo con el idealismo moderno, la labor del espíritu es poner (setzsen). Dado que se concibe el espíritu como sujeto, y es representado así en el esquema sujeto-objeto, el "poner" (tesis) debe ser la síntesis entre el sujeto y sus objetos. Lo que está "puesto" así da una visión de los objetos en su totalidad. Lo que produce la fuerza del sujeto, lo que "pone" entre sí y los objetos merced a la labor, lo denomina Humboldt un "mundo". En semejante "visión del mundo" una humanidad particular alcanza su propia expresión (Heidegger, "El camino al habla", 223-224).

Humboldt contempla el habla como mundo y visión del mundo, dice Heidegger, porque su camino al habla no está determinado desde el habla en cuanto tal, sino que lleva hacia el hombre, conduce a la fundamentación y representación del de-

${ }^{6}$ Bajtín / Voloshinov: "Se puede decir que toda la lingüística posterior a Humboldt, hasta nuestros días, se encuentra bajo su influjo determinante" (Voloshinov, Marxismo y filosofía del lenguaje, 77), Heidegger: desde que se publicó el ensayo Sobre la diversidad de la estructura del lenguaje humano y su influencia sobre el desarrollo espiritual de la humanidad, ese texto "en el pro y en el contra, nombrado o silenciado, determina toda la ciencia lingüística y filosófica que le ha seguido hasta el día de hoy" (Heidegger, "El camino al habla", 183). 
sarrollo espiritual de la especie humana. Y parte de una esencia empobrecida de lo humano, que interpreta la experiencia como una relación lingüística entre el "trabajo del espíritu”, propio del sujeto, y un mundo. Pese a que la interpretación de Heidegger no sea del todo justa con los textos de Humboldt, ${ }^{7}$ debe reconocerse que el "giro lingüístico"8 de este último no logró desplazar completamente hacia la reflexión sobre el lenguaje el problema del sujeto. Este desplazamiento, en cambio, sí se logra en la filosofía del lenguaje de Bajtín, como se verá más adelante.

Él, por su parte, encuentra que el problema más importante del subjetivismo individualista, y con ello de la filosofía del lenguaje de Humboldt, es la ausencia de un enfoque sociológico desde el cual pensar al lenguaje y al sujeto. Y ciertamente, no lo hay.

${ }^{7}$ Difícilmente podríamos discutirle a Heidegger que la investigación humboldtiana sobre el lenguaje sea antropocéntrica. Sin embargo, sí puede discutírsele la interpretación del "trabajo del espíritu" como pura actividad de un sujeto de conocimiento. Si bien hay fragmentos de los escritos sobre el lenguaje de Humboldt que invitan a esta interpretación, algunos otros permiten salir de ella, como el siguiente: "En la lengua se genera un acervo de palabras y un sistema de reglas que, con el paso de los milenios, hacen de ella un poder autónomo. En los capítulos anteriores se ha llamado la atención sobre el hecho de que la idea recogida en el lenguaje se vuelve un objeto para el alma y ejerce por ello una influencia externa sobre ella. Sin embargo, hemos estado considerando el objeto sobre todo como nacido del sujeto, y su efecto como procedente de aquello sobre lo que revierte. Ahora se impone la consideración opuesta, esto es, la de que el lenguaje es realmente un objeto extraño [fremd], y que su efecto procede de hecho de algo distinto de aquello sobre lo que se ejerce. Pues el lenguaje tiene que pertenecer por fuerza a dos, y es en verdad propiedad del conjunto de la especie humana [...] el lenguaje llega a darse a sí mismo una existencia peculiar, que por cierto alcanza vigencia sólo en cada acto de pensar, pero que en su totalidad es independiente de éste. Las dos perspectivas mostradas aquí como opuestas, la de que el lenguaje es extraño al alma y no obstante perteneciente a ella, a un tiempo independiente y dependiente de ella, vienen a unirse realmente en él, y son lo que constituye la peculiaridad de su esencia" (Humboldt, Sobre la diversidad, 86).

${ }^{8}$ Entendido como el reconocimiento de que el lenguaje no es un instrumento para la designación de entidades independientes de él, o para la comunicación de pensamientos anteriores al lenguaje, sino más bien el medio en el cual se constituyen tanto el pensamiento humano (en el sentido en que el lenguaje es la condición de posibilidad, en el sentido kantiano, del pensamiento) como el mundo en donde habitamos (en el sentido en que sólo gracias al lenguaje éste se vuelve interpretable) (Cfr. Lafont, La razón como lenguaje, 15 y ss.). 
Pese a que Humboldt haya considerado la importancia de la historia, la tradición y las culturas singulares para la filosofía del lenguaje, su análisis de las lenguas históricas como lenguas nacionales descartaba lo que Bajtín llamó la estratificación de las lenguas y también otros lenguajes socio-ideológicos. Lo que este último recuperó de la filosofía romántica del lenguaje es la idea de que la lengua está en proceso de generación continua. Una frase muy citada de Humboldt lo dice así: "El lenguaje mismo no es una obra acabada [Werke] (érgon) sino una actividad (enérgeia). Por eso su verdadera definición no puede ser sino genética [genetische]. Pues él es siempre el reiterado trabajo del espíritu de hacer posible que el sonido articulado se convierta en expresión del pensamiento" (Humboldt, Sobre la diversidad, 65). Para Bajtín también el lenguaje está siempre en proceso de generación, sólo que dicha generación no surge del "trabajo del espíritu", sino de la interacción discursiva social de los hablantes (Voloshinov, Marxismo y filosofía del lenguaje, 137).

Con esto llegamos a la segunda característica de la conciencia en Bajtín: la conciencia, además de ser un fenómeno lingüístico e ideológico, es un fenómeno social; o mejor, por ser un fenómeno lingüístico e ideológico, es un fenómeno social.

El signo sólo puede surgir en un territorio interindividual, territorio que no es "natural" en el sentido directo de esta palabra; el signo tampoco puede surgir entre dos homo sapiens. Es necesario que ambos individuos estén socialmente organizados, que representen un colectivo: sólo entonces puede surgir entre ellos un medio sígnico (semiótico). La conciencia individual no sólo es incapaz de explicar nada en este caso, sino que, por el contrario, ella misma necesita ser explicada a partir del medio ideológico social (Voloshinov, Marxismo y filosofía del lenguaje, 33).

Así, la conciencia individual es producto de las relaciones que se dan al interior de una colectividad. Se ha discutido mucho acerca de las posibilidades políticas de esta concepción de la 
construcción de sujetos. Si por "sociedad" se entiende la mera agregación de individuos, y por "interacción discursiva" el diálogo entre una conciencia y un "otro", la problemática se despolitiza. ${ }^{9}$ Sin embargo, hay en El marxismo y la filosofía del lenguaje indicaciones que permiten pensar lo social de otro modo. No solamente la famosa frase sobre que el lenguaje es "la arena de la lucha de clases", sino aquellos momentos en que se refiere al problema de los géneros discursivos como pertenecientes a "cada época y cada grupo social" y a las formas de la comunicación discursiva como "plenamente determinadas por las relaciones de producción y por la formación políticosocial" (Voloshinov, Marxismo y filosofía del lenguaje, 33). Y si la conciencia individual se produce tal como lo permiten las relaciones conflictivas entre grupos sociales, en sus diferentes géneros discursivos, entonces la conciencia es también el lugar del conflicto y no es posible hablar de conciencias, ni de sujetos, que no estén en constante proceso de modificación.

\section{Pluridiscursividad y sujeto}

Uno de los textos de Bajtín que permite pensar de manera más contundente en el lenguaje y también en el sujeto como hábitat de los conflictos sociopolíticos es La palabra en la novela. En el texto es posible encontrar tanto una teoría de la novela como

${ }^{9}$ Y el trabajo del Círculo de Bajtín permite, en momentos, esta interpretación, como lo anota Ken Hirschkop. En "Bakhtin, Discourse and Democracy" escribe: "El problema no es el individualismo, puesto que Bajtín admite que los sujetos pueden ser colectivos o continuos a través de la historia. El problema es que Bajtín define el contexto social únicamente en términos de una multiplicidad de conciencias. En todas sus diversas formulaciones de las relaciones sociales que determinan el estilo, las relaciones son completamente intersubjetivas. La palabra es dialógica en el sentido en que dentro de su ámbito los imperativos ideológicos de cuando menos dos conciencias se intersectan. Pero la situación social se reduce entonces a la interacción entre el sujeto hablante y los otros sujetos" (203). 
una filosofía del lenguaje en la cual la primera se inscribe. Aquí, refiriéndose nuevamente a la filosofía del lenguaje de Humboldt, Bajtín dirige su atención a su nacionalismo lingüístico, o a su definición de las lenguas históricas, singulares, como lenguas nacionales. En la Alemania de las primeras décadas del siglo XIX, dicha definición respondía a un programa político, el de su consolidación como estado-nación. En un sentido mucho más general, según Bajtín, la filosofía del lenguaje de Humboldt pertenecía al grupo de la poética de Aristóteles, la de Agustín, la poética eclesial medieval, la poética de la expresión cartesiana del neoclasicismo, la "gramática universal" de Leibniz, y respondía, como todas ellas, a las fuerzas centralizadoras del universo ideológico-social y cultural. Estas fuerzas históricas, que Bajtín metaforiza como "fuerzas centrípetas", sirven al objetivo "de centralización y unificación de las lenguas europeas", y la categoría del "lenguaje único" es su expresión teórica. La eficacia de estas fuerzas se muestra de distintas maneras: en primer lugar, por su capacidad de imposición. En efecto, el lenguaje único no viene dado sino que se impone siempre: se impone una lengua predominante, se eliminan otras, se educa a través de la lengua oficial, se canonizan los sistemas ideológicos, etc. En segundo lugar, es una fuerza sumamente eficaz como garante del "máximo entendimiento recíproco" que proporciona la lengua como "visión del mundo" en el sentido de Humboldt.

Las fuerzas centrípetas de las lenguas se oponen a, y se imponen sobre, la pluridiscursividad del lenguaje. Bajtín considera que "la lengua, en cada uno de los momentos de su proceso de formación, no sólo se estratifica en dialectos lingüísticos en el sentido exacto de la palabra sino también - y ello es esencialen lenguajes ideológico-sociales: de grupos sociales, 'profesionales', 'de género', lenguajes de las generaciones, etc.” (Bajtín, "La palabra en la novela", 89). Estas fuerzas, que viven en la novela y en los géneros literarios en prosa, en las "capas bajas" 
de la sociedad, en los "escenarios de las barracas y las ferias", en las calles, etc., se orientan, en la historia europea, contra los lenguajes oficiales contemporáneos: están dialogizadas. ${ }^{10}$ Para Bajtín, la novela y la prosa en general pueden constituir el lugar privilegiado de dialogización de los lenguajes ideológicosociales. Pero fuera del ámbito de la novela, estos lenguajes se dialogizan en general en el uso de la palabra, en la vida del lenguaje. Est dialogización está determinada "por la lucha entre puntos de vista socio-lingüísticos y no por el conflicto intralingüístico de las voluntades individuales o de las contradicciones lógicas" (Bajtín, "La palabra en la novela", 91), lo cual es de la mayor importancia para la construcción del sujeto, como veremos.

Las fuerzas centrípetas y centrífugas del lenguaje tienen un equivalente cuando el análisis se refiere a las relaciones entre la palabra y el hablante: las primeras corresponden a la palabra autoritaria, y las segundas a la que Bajtín llama palabra intrínsecamente convincente. Se trata de las formas que puede tomar la palabra ajena, que es el material con el cual se construye la conciencia individual. La palabra autoritaria es la que carece de persuasión intrínseca; formalmente, requiere cierto distanciamiento por parte del hablante, no es la clase de palabra que pueda ser asimilada por él, en el sentido bajtiniano de "decir algo con las propias palabras" (por oposición a "repetir de memoria"); se trata de aquella palabra cuya estructura semántica está cerrada o calcificada. Esta clase de discurso da lugar al autoritarismo, al tradicionalismo, al universalismo, a lo oficial, al poder político e institucional. Por otra parte, la palabra internamente persuasiva no está vinculada a ninguna clase de

\footnotetext{
${ }^{10}$ Las relaciones dialógicas, que para Bajtín son propias de la palabra, es decir, de la lengua "como fenómeno total y concreto", no pueden reducirse a relaciones lógicas ni temático-semánticas. Son, para él, todas las relaciones posibles entre enunciados, entre "posiciones" de diferentes sujetos (Bajtín, Poética de Dostoievski, 267).
} 
autoridad, puede ser asimilada por el hablante y es fundamentalmente productiva: da lugar a nuevo pensamiento y a nuevos discursos. Su estructura semántica es abierta.

La conciencia puede pensarse como el lugar donde se producen las complejas relaciones dialógicas, a menudo conflictivas y a veces francamente antagónicas, entre todas las fuerzas socio-ideológicas que se manifiestan en el discurso ajeno. Relaciones conflictivas entre discursos autoritarios y discursos no autoritarios, y entre los diversos géneros de discursos no autoritarios entre sí, que pueden dar lugar a un sujeto en proceso permanente de construcción, y que por tanto es capaz de poner en cuestión una identidad determinada socio-ideológicamente. Gracias a las fuerzas del discurso puede ser un sujeto que cuestione los lugares, las funciones y las prácticas que un cierto régimen político le haya asignado. Se trata de un sujeto discursivo. Pero esto implica que la dialogización de los discursos en la conciencia individual, tal como en el uso de la palabra, depende de la lucha entre puntos de vista socio-ideológicos y no de la lucha entre voluntades individuales, y que la posibilidad de cuestionamiento tampoco depende de una voluntad individual, sino del estado de las fuerzas discursivas; es decir, de factores políticos, sociales, culturales, etc., y del emplazamiento del sujeto en un lugar y en un momento determinados al interior de estas fuerzas.

Bajtín usa el caso hipotético de un campesino para explicar cómo se ponen en relación las fuerzas discursivas en una subjetividad específica. En él conviven distintos lenguajes: aquel en el que reza, otro en el que canta, otro en el que habla con la familia, y un cuarto, en el que formula sus demandas a las autoridades locales. Se puede pasar de uno a otro de estos lenguajes heterogéneos de forma automática, sin poner en cuestión el uso de cada uno, por costumbre. Pero llega el momento cuando es posible mirar uno de estos lenguajes y al universo discursivo que le corresponde con los ojos de otro. Con ello, estos univer- 
sos diferentes y hasta contradictorios se ponen mutuamente en cuestión. Es posible pensar en el proceso de construcción del sujeto de la misma manera.

Así, la posibilidad de cuestionar las prácticas discursivas que definen una subjetividad específica al asignarle espacios y tiempos, y también formas de actividad, sucede cuando las fuerzas discursivas lo permiten, cuando entran en conflicto. Pienso que esta concepción del sujeto discursivo puede ser muy útil para explicar la emergencia (o por el contrario, la ausencia) de los diversos movimientos políticos de resistencia. Para volver al planteamiento inicial, puede también explicar la manera como las víctimas de exclusión en nuestros sistemas políticos hablan de la construcción de subjetividades alternativas. Así lo explica Nagar-Ron refiriéndose al caso de las mujeres Misrahi. De acuerdo con ella, "la experiencia de sentirse avergonzada del propio origen" acompaña a la primera etapa de la conciencia étnica. Como se dijo, ésta se caracteriza por la internalización de las percepciones hegemónicas socialmente prevalecientes y por el desarrollo de una personalidad que se ajusta a estas percepciones. El siguiente testimonio de Lilian (de 50 años, doctora en ciencias políticas), quien nació en Marruecos y emigró con su familia a Israel cuando tenía tres años de edad, es un ejemplo de dicha "internalización”:

A partir de la edad de diez años, aproximadamente, recuerdo que yo misma trabajaba sobre mi acento, para no hablar con la Het y la Ayin guturales. Mentía y decía que era francesa. Pero eso requería de un mundo absolutamente diferente. En primer lugar - no le permitía a mi madre que hablara árabe en la calle; nunca invitaba a mis amigos a la casa, para que no se dieran cuenta. Construí un mundo imaginario [...] fui con mi madre para que me contara la historia de Francia [...] desde sexto año realmente viví una mentira (Nagar-Ron, "Identidad, resistencia y voz", 18). 
En términos bajtinianos, esta internalización puede explicarse fácilmente desde la perspectiva del discurso autoritario. En este caso es el discurso "orientalista", que marca a Oriente como el Otro y desde esta alteridad establece su identidad Occidental, como lo anota Nagar-Ron siguiendo a Edward Said y a Ella Shohat. La segunda etapa de la "conciencia de sí" se relaciona, como se dijo, con el proceso mediante el cual las mujeres tomaron conciencia de la opresión que habían sufrido como parte de un grupo excluido, y con los cambios experimentados en la conciencia de sí y en la identidad como resultado de esta nueva toma de conciencia. Estos cambios fueron posibles, según Nagar-Ron, gracias al contacto con un "agente de conocimiento". En este caso queda personificado por Ella Shohat, renombrada feminista Misrahi, profesora de estudios culturales de la Universidad de Nueva York, quien participó en talleres auspiciados por varias organizaciones sociales. Lo que el "agente de conocimiento" ofrece es una clase de discurso no autoritario, en el sentido de Bajtín, capaz de poner en cuestión una subjetividad socialmente construida. Así lo explica Nagar-Ron:

El cambio de conciencia experimentado por las mujeres después del encuentro con un agente de conocimiento les permite conceptualizar sus sentimientos y emplear intuiciones intelectuales para hacer coherente su propia identidad. Ellas entienden que hasta ahora podían haber sido aceptadas en la esfera pública como "Israelitas" — en otras palabras, occidentales avanzadas, siempre y cuando escondieran su etnicidad. Ahora ellas se levantan frente a la fragmentación entre los diferentes aspectos de su identidad. La posibilidad de coherencia entre el "yo" privado y el "yo" público las conduce a llevar a cabo actos sociales y subversivos, que tienen como propósito exponer la conciencia orientalista que yace en la base del establecimiento del Estado y la sociedad de Israel ("Identidad, resistencia y voz", 20).

Los aspectos de la propia identidad fragmentada de los cuales se habla aquí vienen dados en distintos discursos socio-ideo- 
lógicos. Más que hablar de "conciliación" o de "identidad", términos de los cuales la filosofía de la diferencia nos ha enseñado a desconfiar, quisiera subrayar que el análisis sociológico de Nagar-Ron muestra cómo estos sujetos, gracias a las relaciones discursivas dialógicas, fueron capaces de poner en cuestión las prácticas de subjetivación impuestas por las políticas hegemónicas. En este caso el testimonio de los excluidos es una forma del discurso privilegiada para la dialogización, y por lo tanto, un terreno experimental para nuevas prácticas de subjetivación.

\section{REFERENCIAS}

BAJTín, Mijaíl, "La palabra en la novela", en Teoría y estética de la novela, trad. H. S. Kriúkova y V. Cazcara, Madrid, Taurus, 1989, 77-236.

_- Problemas de la poética de Dostoievski, trad. Tatiana Bubnova, México, Fondo de Cultura Económica, 2005.

Bubnova, Tatiana, "Bajtín en la encrucijada dialógica (datos y comentarios para contribuir a la confusión general)", en Bajtín y sus apócrifos, Iris Zavala (coord.), México, Anthropos, Editorial de la Universidad de Puerto Rico, 1996, 13-72.

Gardiner, Michael, M. M. Bakhtin and the Theory of Ideology, London-New York, Routledge.

HeidegGer, M., "El camino al habla", en De camino al habla, Barcelona, Ed. del Serbal, 2002, 177-200.

HiRschKop, Ken, "Bakhtin, Discourse and Democracy", en Gardiner, Mikhail Bakhtin, vol. III, Michael E. (comp.), London, Thousand Oaks, New Delhi, sage Publications, 2003, 92-113.

Humboldt, W., Sobre la diversidad de la estructura del lenguaje humano y su influencia sobre el desarrollo espiritual de la humanidad, Barcelona, Anthropos, 1990.

LAFONT, Cristina, La razón como lenguaje, Madrid, Visor, 1993.

Martínez de la Escalera, Ana María, "Prólogo" a Estrategias de resistencia, Ana María Martínez de la Escalera (coord.), México, Universidad Nacional Autónoma de México, PUEG, 2007, 7-9. 
NAGAR-Ron, Sigal, "Identidad, resistencia y voz: ser una mujer judía oriental y feminista en Israel", en Ana María Martínez de la Escalera (coord.), Estrategias de resistencia, México, Universidad Nacional Autónoma de México, PUEG, 2007, 15-20

Rancière, Jacques, The Politics of Aesthetics. The Distribution of the Sensible, London, Continuum, 2004.

Voloshinov, Valentin N, El marxismo y la filosofía del lenguaje (Los principales problemas del método sociológico en la ciencia del lenguaje), trad. Tatiana Bubnova, Madrid, Alianza, 1992. 